Article

\title{
Automatic Lab on PCB system for agarose gel preparation and electrophoresis for biomedical applications
}

\author{
Jesús David Urbano-Gámez ${ }^{1}$, Francisco Perdigones ${ }^{1}\left[\right.$, and José Manuel Quero ${ }^{1} \mathbb{C}$ \\ 1 Electronic Engineering Department, University of Seville
}

\begin{abstract}
In this paper, a prototype of an automatic lab on PCB for agarose preparation and electrophoresis is developed. The dimensions of the device are $38 \times 34 \mathrm{~mm}^{2}$ and includes a conductivity sensor for detecting the TAE buffer (Tris-Acetate-EDTA buffer), a microheater for mixing, a NTC thermistor for controlling the temperature, a LDR sensor for measuring the transparency of the mixture, and two electrodes for performing the electrophoresis. The agarose preparation functions are governed by a microcontroller. The device requires a PMMA structure to define the wells of the agarose gel, and to release the electrodes from the agarose. The maximum voltage and current that the system requires are $40 \mathrm{~V}$ to perform the electrophoresis, and $1 \mathrm{~A}$ for activating the microheater. The chosen temperature for mixing is $80^{\circ} \mathrm{C}$, with a mixing time of $10 \mathrm{~min}$. In addition, the curing time is about $30 \mathrm{~min}$. This device is intended to be integrated as a part of a larger lab on PCB system for DNA amplification and detection. However, it can be used to migrate DNA amplified in conventional thermocyclers. Moreover, the device can be modified for preparing larger agarose gels and performing electrophoresis in an automatic manner.
\end{abstract}

Keywords: Lab on PCB; Electrophoresis; Biomedical applications; Agarose

\section{Introduction}

Nowadays, the development of microfluidic devices using printed circuit board (PCB) substrates has been the subject of increasing research [1,2]. These devices are named lab on PCBs, and they can be considered as a part of lab on a chip devices (LoCs). The use of PCB substrates has interesting advantages for biomedical applications [3], such as the possibility of an easy integration of electronics and microfluidics with sensors and actuators in a single platform; commercial availability and low cost production, to name a few. It is important to emphasise that lab on PCBs can include several laboratory tasks, such as micromixing, sensing, chemical reactions and heating in a device with the dimensions of a credit card.

Apart from the PCB substrate, lab on PCBs have been fabricated using different rapid prototyping materials, for example SU-8 [4,5] and PDMS [6,7]. However, the use of thermoplastics for the microfluidic component of the device is a more interesting option from the point of view of the market. In this respect, the device fabrication can be intended as a mass production, that is, the thermoplastic fabrication using hot embossing or injection molding, and the PCB can ordered to specialised companies. These characteristics make lab on PCBs an attractive choice due to their high potential of commercialization [1,2].

Up to now, lab on PCBs include several biomedical applications, for example, for detecting cell viability [8], molecular diagnosis [9], organotypic cultures [10], or electrolytes detection [11]. Focusing on electrophoresis-based applications, capillary electrophoresis (CE) is the most usual technique in lab on chip, [12]. This is because this kind of electrophoresis may be automated and direct quantification 
is possible. Among others, CE has been used for separation of monosaccharides, oligosaccharides, and polysaccharides [13], separation of proteins [14] and for life science in general [15]. On the other hand, conventional electrophoresis continues being a robust and very used method [16] both for lab on a chip applications $[17,18]$ and conventional laboratories [19].

Whatever the method, the electrophoretic gel (agarose or polyacrylamide) is manually fabricated in many laboratories. For instance, agarose gel electrophoresis is typically used to resolve RNA and DNA; and polyacrylamide gel electrophoresis is used to separate proteins. These methods require heating, time to achieve transparency and a curing time to solidified the material. In addition, the integration of the conventional electrophoretic method on lab on chip is challenging due to the low automatization of the method. Moreover, the quantity of agarose for both LoC and large laboratories is limited to the commercial electrophoretic tanks dimensions. In this respect, new techniques have been used for preparing the agarose gel [20].

In this paper, an automated lab on PCB for preparing agarose gel is described. It includes a microheater to reach the mixing temperature of agarose and TAE buffer (Tris-Acetate-EDTA buffer). Regarding the buffer, TBE buffer (Tris-Borate-EDTA buffer) could be used as well. The micro-platform has integrated sensors, such as thermistor to control the temperature and light-dependent-resistor to ensure the required transparency. In addition, it includes an interdigitated capacitive sensor to detect the filling of the cavity with the liquid. All these sensors and actuators are connected to a microcontroller with governs the working of the lab on PCB. The device testing consists in fabricating the agarose gel using the device, and perform the electrophoresis. The device can be intended as an integrable functional module of a more complex lab on $\mathrm{PCB}$, or even a device itself to perform electrophoresis in conventional laboratories.

\section{Lab on PCB brief description}

In this section the working of the lab on PCB is briefly described. Then, in Section 3, the sensors and actuators are described for a complete understanding of the device.

The PCB substrate has two functional components, Figure 1. The first one is intended to control the agarose mixing and curing, and the second one for performing the electrophoresis. The dimensions of the PCB are $38 \times 34 \mathrm{~mm}^{2}$.

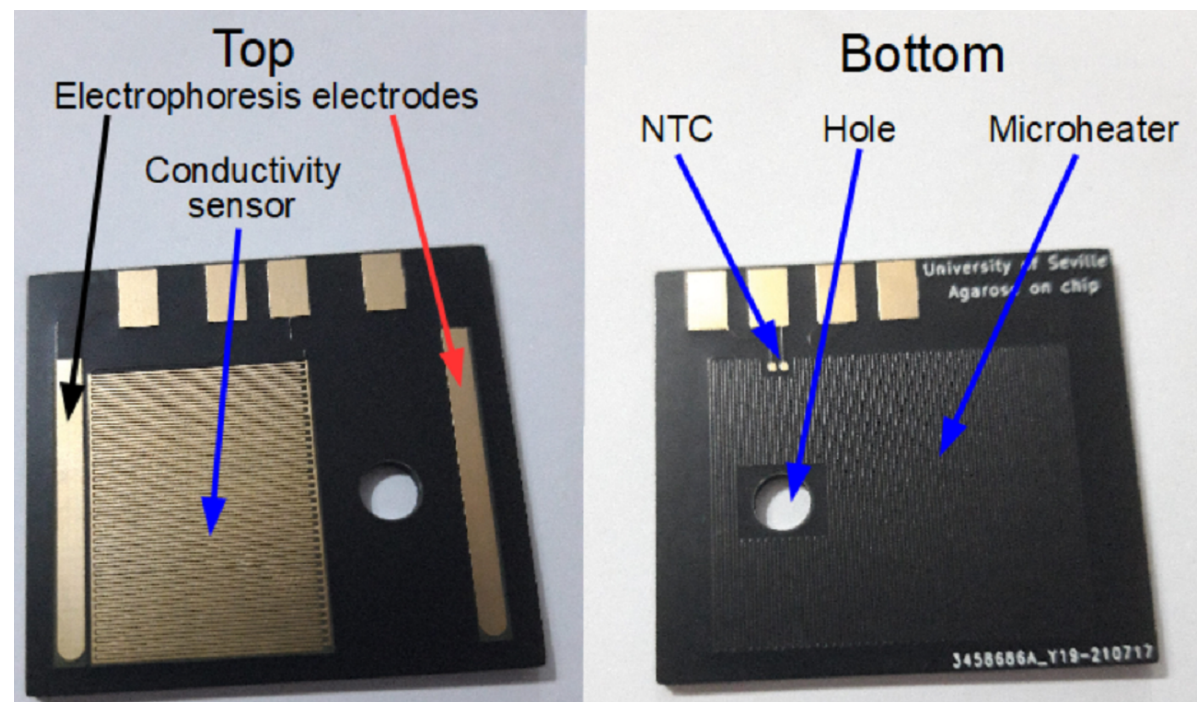

Figure 1. Printed Circuit Board substrate for agarose mixing and curing, and electrophoresis. Left: Conductivity sensor and electrophoresis electrodes are shown. Right: The microheater and the thermistor can be seen. The dimensions of the device are $38 \times 34 \mathrm{~mm}^{2}$

Regarding the preparation of the agarose, the device includes a microheater in order to mix the agarose with TAE buffer. Typically, the mixing is considered finished when the agarose is 
transparent enough. Therefore, the degree of transparency needs to be monitored. In order to do so, a Light-Dependent-Resistor (LDR) sensor is used. In addition, the mixing temperature is measured with an integrated Surface Mounted Device (SMD) thermistor to perform the control of temperature. On the other hand, the device includes a conductivity sensor in order to detect the filling of the cavity with the liquid to start the automatic process. Theses steps, that is, the mixing and the curing of the agarose is governed by a microcontroller. Finally, the electrophoresis is performed using two gold electrodes integrated in the same PCB substrate.

The device has polymethylmethacrylate (PMMA) walls which limit the area of the fabricated agarose gel. This part has an auxiliary structure to define both the wells to charge the DNA and the volume above the electrodes to store the TAE buffer, Figure 2. The auxiliary structure has to be inserted in the cavity before the filling with the TAE buffer.

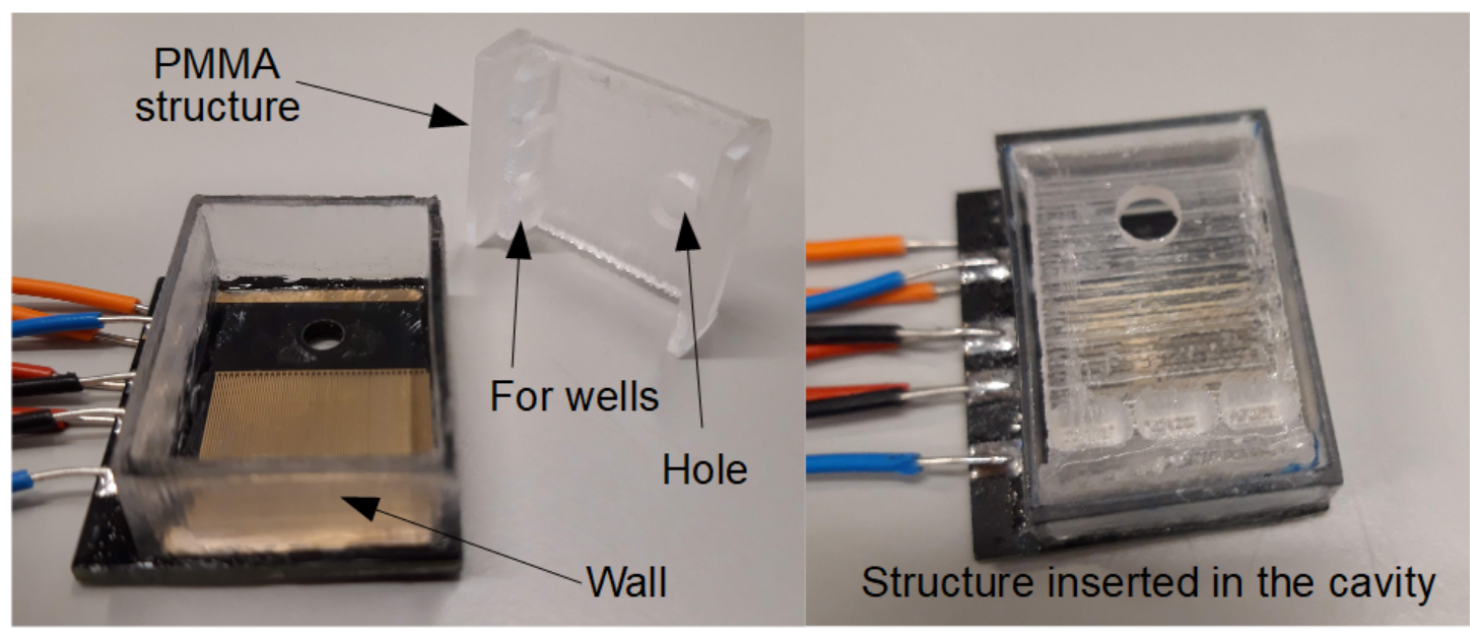

Figure 2. The lab on PCB with the thermoplastic wall and the auxiliary structure are shown

The device requires a basic signal condition circuit in order to manage both the current along the microheater and the measure of the sensors outputs. The whole behaviour of the lab on PCB is governed by a microcontroller.

\section{Sensors and actuators description}

\subsection{Thermal actuation}

The microheater is directly integrated in the bottom side of PCB substrate during fabrication, and covered with the solder mask. It is performed by the commercial PCB manufacturer with a very good finish. This microheater is a copper serpentine with a length of $2.4 \mathrm{~m}$, a width of $150 \mu \mathrm{m}$, a spacing between lines of $150 \mu \mathrm{m}$, and a thickness of $35 \mu \mathrm{m}$. The experimental resistance of the copper microheater is $7 \Omega$.

The control of the temperature is performed using a SMD thermistor (Part number: NXFT15XH103. Resistance $=10 \mathrm{k} \Omega$, B-constant $=3380 \mathrm{~K}$ and package 0402, Murata Manufacturing Co., Ltd.) which is placed close the microheater. The temperature need to be characterized previously because the temperature of the liquid over the microheater and the thermistor temperature is not necessarily the same. This characterization is described in Section 5.

\subsection{Optical sensing}

The PCB substrate has a hole with a diameter of $4.5 \mathrm{~mm}$, covered with a transparent and PCR-compatible film (ThermalSeal $®$ films, classic). The optical sensor is a LDR (NSL-19M51) with a diameter of $4.3 \mathrm{~mm}$ and a minimum light resistance of $20 \mathrm{k} \Omega$. It is placed just below the transparent film of the PCB hole. As can be seen in Figure 2, the auxiliary structure has a hole to avoid the lack of 
transparency due to the CNC laser fabrication process. This hole is just above the PCB hole in order to create an optical path which allows the measure of the transparency. The values of the optical sensor have to be characterized to define the transparency at the end of both the mixing and the curing. This characterization is described in Section 5.

\subsection{Conductivity sensing}

Likewise the microheater, the conductivity sensor is fabricated by the manufacturer company, in this case in the top side of the substrate and covered with gold. This sensor is an integrated interdigitated transducer (IDT) with 74 electrodes with a width of $150 \mu \mathrm{m}$, a gap of $150 \mu \mathrm{m}$, and a length of $1.8 \mathrm{~cm}$. The dimensions of the electrodes and the microheater are limited by the selected technology, but they can be reduced by increasing the fabrication cost. This sensor requires characterization in order to define the value for detecting the TAE buffer. This characterization is described in Section 5 together the optical and thermal experiments.

\subsection{Electrophoretic actuation}

The migration of the DNA is performed actuating on the lateral electrodes. These electrodes were integrated during the fabrication process. They are copper electrodes covered with gold for reducing the oxidation during the electrophoresis process. The electrodes have a length of $2.8 \mathrm{~cm}$, a width of 2 $\mathrm{mm}$ and a thickness of $35 \mu \mathrm{m}$.

\section{Process sequence}

The steps of the process can be seen in table 1 . These steps are going to be commented in detail in the following.

Table 1. Whole process sequence for agarose gel preparation and electrophoresis

\begin{tabular}{ccc}
\hline \multicolumn{3}{c}{ Agarose fabrication } \\
\hline Step & Function & Automatic \\
\hline 1 & Filling of the cavity & Yes \\
2 & Mixing & Yes \\
3 & Curing detection & Yes \\
4 & Removing of the auxiliary structure & No \\
\hline \multicolumn{3}{|}{ Electrophoresis } \\
\hline Step & Function & Automatic \\
\hline 5 & DNA loading & No \\
6 & Electrophoresis & No \\
\hline
\end{tabular}

The device with the PMMA structure assembled can include the agarose powder (CSL-AG500 Cleaver Scientific) over the surface before starting the process, in this case $100 \mathrm{mg}$ for a $4 \mathrm{ml}$ agarose gel (final concentration 2,5\% w/v). The first step consists in filling the cavity with the agarose-TAE mixture with SYBRSafe DNA staining solution (S33102 ThermoFisher Scientific) to perform the mixing, where the TAE buffer is (Ref. 15558042 ThermoFisher Scientific). This filling is performed using a syringe pump (NewEra Pump Systems NE-1000), with a volume of $4 \mathrm{~mL}$. The percentage of agarose can be modified by changing the TAE buffer volume, the quantity of agarose or both of them. This step is detected by the conductivity sensor, which sends the signal for starting the automatic process. The next step consists in disabling the conductivity sensor, supplying the required current to the microheater, and sensing the degree of transparency. Once the transparency is achieved the third step takes places automatically, that is, the microheater is disabled in order to cool down the mixture. In this step the LDR sensor continues enabled because the degree of transparency of the cured agarose is lower than the freshly mixed agarose. The automatic process finishes when the agarose is cured, after 
which the microcontroller activates a LED in order to inform that the process is finished, and disable the sensors. Finally, the following step is not automatic, it consists in removing the PMMA structure to define both the wells and the cavities to pour the TAE buffer. After this process, the device is ready to be loaded with the liquid to be migrated using electrophoresis. The experimental results will shown the performance of the fabricated agarose gel for electrophoresis.

Once the agarose gel is cured and the PMMA structured is removed from the substrate, the next step consists in loading the wells with DNA. After that, the electrophoresis starts, in order to do so, the lateral gold electrodes are used. In this case, these two steps are manually performed.

It is worth highlighting that the filling process could be performed manually, or even integrated in the automatic process. The last option would imply that the conductivity sensor would not be necessary for detecting the liquid. In addition, the mixing temperature and the required degree of transparency could be modified if necessary. Finally, although the electrophoresis process is disconnected to the agarose fabrication, they can be joined by software. In order to do, automatic pipettes, and the integration of the actuation of the lateral electrodes are necessary. This is possible, but the low cost nature of the device is lost. However, it could be interesting for large laboratories.

\section{Experimental results}

Before performing the experiment to fabricate the aragose gel and define a program to control the process, the characterization of the sensors and actuators is required.

The microheater characterization consists in relating the temperature of the agarose-TAE solution with the temperature of the NTC thermistor. In order to do so, a thermocouple has been used for measuring the temperature of the liquid of the cavity (agarose-TAE solution). In addition, the current supplied to microheater has to be defined. The results for the microheater can be seen in Figure 3.

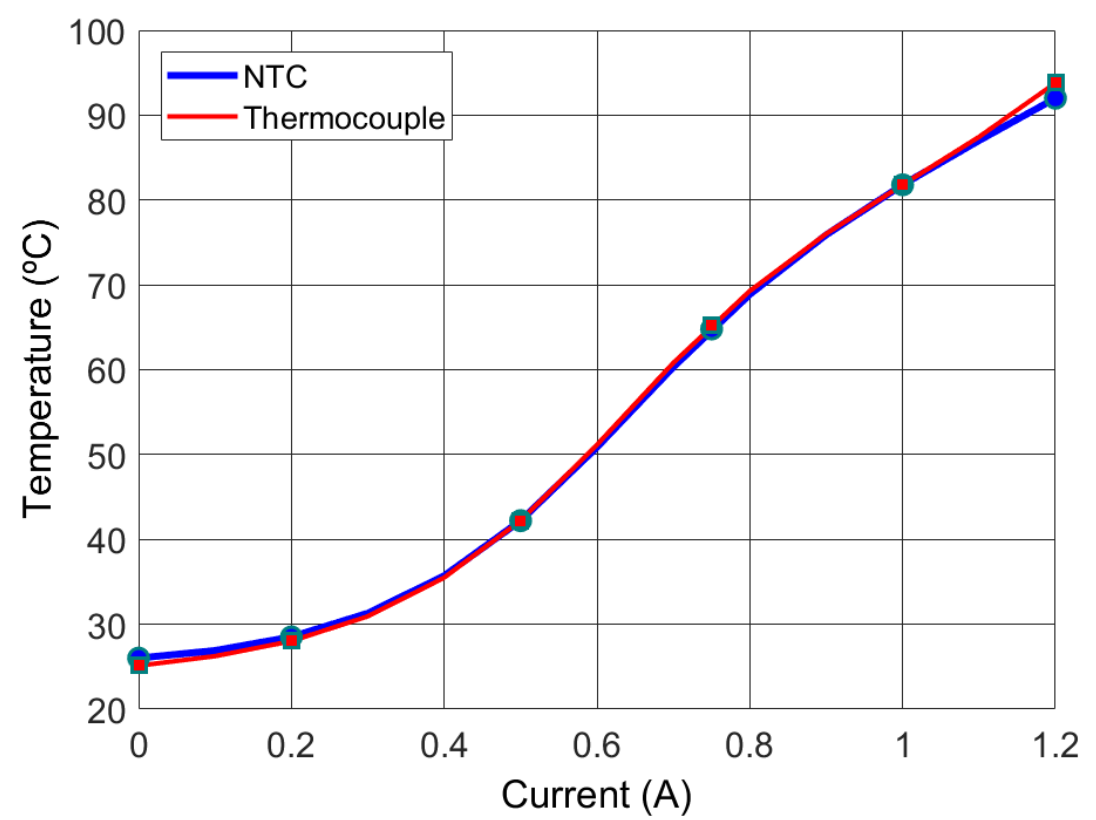

Figure 3. Temperature of both the agarose-TAE solution (thermocouple) and the NTC thermistor as a function of the current

The temperature for performing the agarose gel is chosen to be a set point of $80^{\circ} \mathrm{C}$. This implies a thermistor resistance of $1680 \Omega$. The electronic circuit to control the temperature is a voltage divider, so that the voltage of the thermistor is applied to an analog pin of the microcontroller. In addition, a driver (TC4427CPA) and a power MOSFET (nMOS IRFB4227PBF) are required to supply a controlled current to the microheater. As can be seen, the temperatures of the NTC and the liquid (thermocouple) 
are very close. This is due to the NTC is far from the center of the microheater (Figure 1), and the liquid is on the opposite side of the microheater. The author have considered that the temperatures are the same for this device, however for a different design of the device this assumption could not be correct.

The characterization of the IDT conductivity sensor is simple to perform. In this case, the conductance before filling the cavity with the TAE buffer is $0 \mathrm{~S}$. When the liquid is in the cavity the conductance decreases up to $0,04 \mathrm{mS}$, which corresponds to a resistance of $20 \mathrm{k} \Omega$. This value is chosen to define the starting point of the process, that is, the process starts when the conductivity sensor reachs $0,04 \mathrm{mS}$. Likewise the NTC sensor, the electronic circuit is a voltage divider.

The characterization of the optical sensor consists in measuring the degree of transparency of the agarose-TAE solution. In order to do so, another voltage divider is used, where the voltage of the LDR is measured. In this case, the agarose gel performed is $2,5 \% \mathrm{w} / \mathrm{v}$ in TAE buffer. The choice of the final transparency of the mixture is defined taking into account the expertise of the authors. These results can be seen in Figure 4.

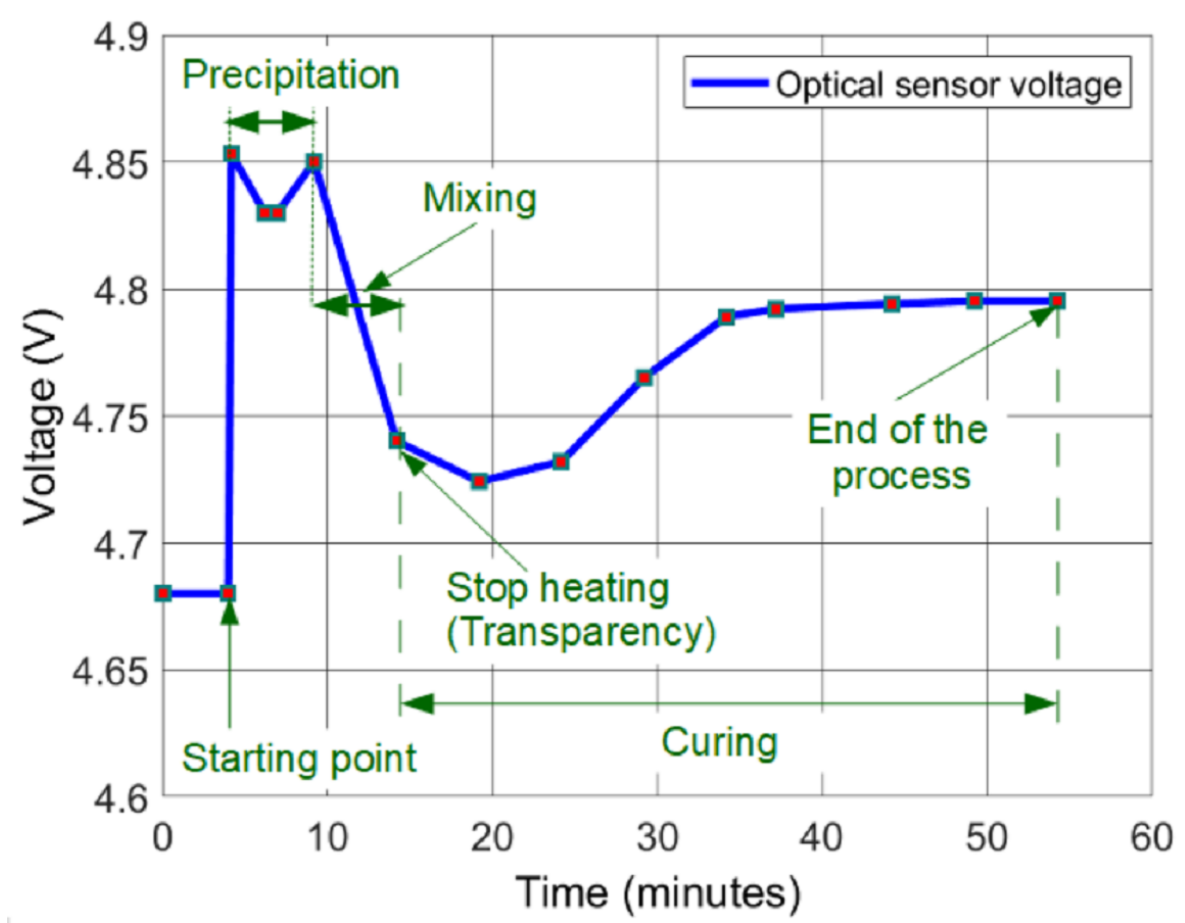

Figure 4. Different values of the optical sensor voltage as a function of the time

The initial value of the optical sensor is $4.68 \mathrm{~V}$ before pouring the TAE buffer. After that, this value increases up to $4.85 \mathrm{~V}$ when the powder goes to the surface of the liquid. Then, the powder starts to precipitate and a slightly transparency is achieved. When the powder finished to precipitate that transparency is lost. At this point the mixing starts and the liquid begins to become transparent. Therefore, the voltage decreases. The final transparency is reached at $4.74 \mathrm{~V}$, and the microheater is disabled. Later on, the liquid continues increasing the transparency during the cooling down. Then, the agarose starts curing. Once the transparency is lost again, the process finishes, V = 4.795 V. All these values have to be taking into account for programming the microcontroller (ATMega380P).

The process for preparing the agarose gel takes $40 \mathrm{~min}$ from the beginning, where the first seconds are used for filling with the TAE buffer, and the last seconds for removing the PMMA structure. The remaining time is automatic, where the mixing time is $10 \mathrm{~min}$ and the curing time is about $30 \mathrm{~min}$.

Once the sensors and actuators are characterized and the microcontroller is programmed, the experiments are carried out. The resulting agarose gel after both the mixing and the curing is shown in Figure 5. In addition, the wells loaded with liquids can be seen. 


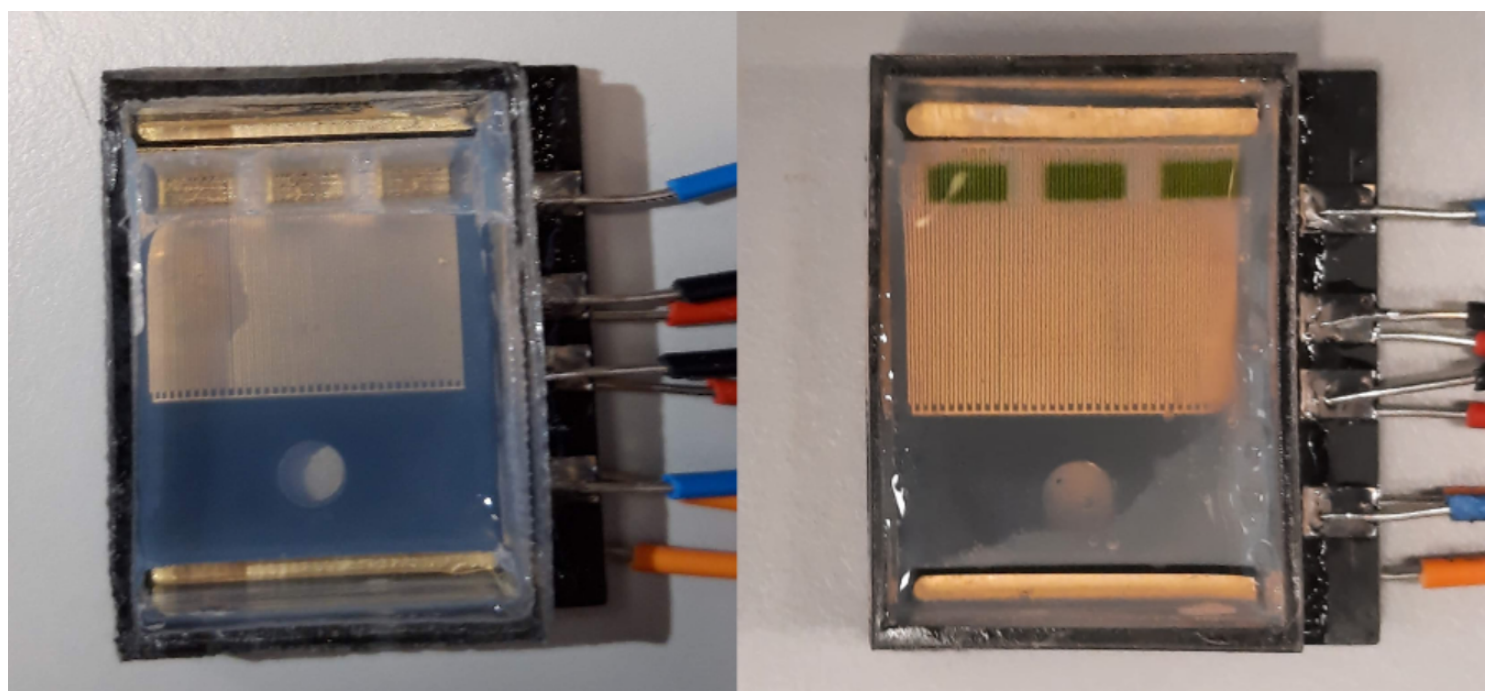

Figure 5. The result of the mixing and curing after removing the structure can be seen. In addition, the weel have been filled with liquids

The device is checked with DNA in order to verify the correct migration of DNA along the agarose gel. This is important to analyse the homogeneity of the agarose gel. In order to do so, the electrophoresis is performed at $40 \mathrm{~V}$ with a required current of $20 \mathrm{~mA}$. The negative and positive electrodes were shown in Figure 1 with black and red arrows, respectively.

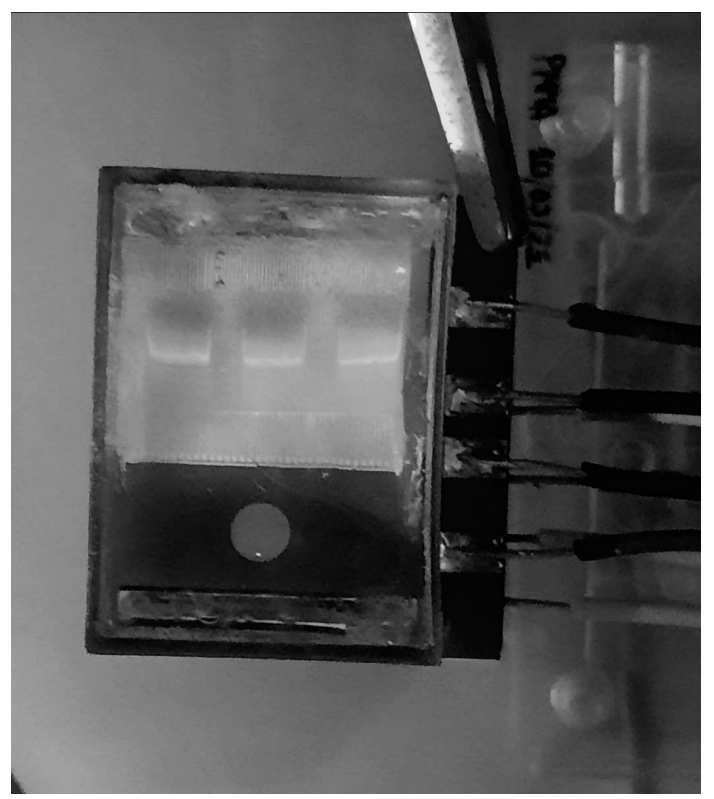

Figure 6. Bands obtained after migrating the DNA by electrophoresis

As can be seen, three parallel bands have been obtained after the migration by electrophoresis. These bands are not deformed in the aragose gel. Therefore, the homogeneity is acceptable.

\section{Discussion and Conclusion}

An automatic prototype of a device for agarose gel preparation, and electrophoresis migration is described. It includes a conductivity sensor for detecting the agarose-TAE solution in order to start the automatic process. In addition, the device has an integrated microheater and a NTC thermistor for controlling the mixing temperature. Moreover, an optical sensor is used for measuring the degree of transparency. The device is fabricated using commercial PCB substrates and thermoplastics. This fact 
implies low cost and mass production fabrication. Furthermore, the inexpensive nature of the device implies disposability.

The proposed lab on PCB is intended to be integrated with lab on chip thermocyclers and fluorescence detection systems for developing automatic PCR devices. The whole automatic system has to include the DNA amplification, the agarose gel fabrication, electrophoresis and detection. The application of these devices is point of care diagnosis of qualitative PCR, and individual uses. Furthermore, the proposed system can be used for conventional PCR procedures. In addition, the device can be fabricated with smaller dimensions for a higher integration on lab on chip, or even larger for conventional multiwells agarose gels.

Author Contributions: “Conceptualization, F.P.; methodology, F.P. and J.D. U-G.; software, F.P.; validation, F.P. and J.D. U-G.; formal analysis, J.D. U-G.; investigation, F.P and J.D. U-G. ; resources, J.M.Q. and F.P.; data curation, F.P. writing-original draft preparation, F.P. and J.D. U-G.; writing-review and editing, J.D. U-G.; visualization, F.P; supervision, J.M.Q. and F.P; project administration, F.P.; funding acquisition, J.M.Q and F.P. All authors have read and agreed to the published version of the manuscript."

Funding: This work has been funded by regional government Junta de Andalucía (Consejería de Economía y Conocimiento), Plan Andaluz de Investigación, Desarrollo e Innovación (PAIDI 2020) with the project "Sistema para la amplificación y detección de fragmentos de ADN empleando PCR en Lab-on-chip (PCR-on-a-Chip)", reference project P18-RT-1745. The authors also thank "Proyectos I+D+i FEDER Andalucía 2014-2020", project "Lab-on-chip de electro-estimulación, para el estudio In-vitro de Cultivos de retina de Larga duración: Retina-on-a-chip" reference project US-1265983

Conflicts of Interest: "The authors declare no conflict of interest."

\section{Abbreviations}

The following abbreviations are used in this manuscript:

MDPI Multidisciplinary Digital Publishing Institute

DOAJ Directory of open access journals

TLA Three letter acronym

LD linear dichroism

\section{References}

1. Perdigones, F. Lab-on-PCB and Flow Driving: A Critical Review. Micromachines 2021, 12, 175.

2. Moschou, D.; Tserepi, A. The lab-on-PCB approach: tackling the $\mu$ TAS commercial upscaling bottleneck. Lab on a Chip 2017, 17, 1388-1405.

3. Zhao, W.; Tian, S.; Huang, L.; Liu, K.; Dong, L. The review of Lab-on-PCB for biomedical application. Electrophoresis 2020, 41, 1433-1445.

4. Flores, G.; Aracil, C.; Perdigones, F.; Quero, J.M. Lab-protocol-on-PCB: Prototype of a laboratory protocol on printed circuit board using MEMS technologies. Microelectronic Engineering 2018, 200, $26-31$.

5. Flores, G.; Aracil, C.; Perdigones, F.; Quero, J. Low consumption single-use microvalve for microfluidic PCB-based platforms. Journal of Micromechanics and Microengineering 2014, 24, 065013.

6. Chang, Y.; You, H. Efficient Bond of PDMS and Printed Circuit Board with An Application on Continuous-flow Polymerase Chain Reaction. BioChip Journal 2020, 14, 349-357.

7. Burdallo, I.; Jimenez-Jorquera, C.; Fernández-Sánchez, C.; Baldi, A. Integration of microelectronic chips in microfluidic systems on printed circuit board. Journal of Micromechanics and Microengineering 2012, 22, 105022.

8. Nikshoar, M.S.; Khosravi, S.; Jahangiri, M.; Zandi, A.; Miripour, Z.S.; Bonakdar, S.; Abdolahad, M. Distinguishment of populated metastatic cancer cells from primary ones based on their invasion to endothelial barrier by biosensor arrays fabricated on nanoroughened poly (methyl methacrylate). Biosensors and Bioelectronics 2018, 118, 51-57.

9. Jolly, P.; Rainbow, J.; Regoutz, A.; Estrela, P.; Moschou, D. A PNA-based Lab-on-PCB diagnostic platform for rapid and high sensitivity DNA quantification. Biosensors and Bioelectronics 2019, 123, 244-250.

10. Cabello, M.; Mozo, M.; De la Cerda, B.; Aracil, C.; Diaz-Corrales, F.J.; Perdigones, F.; Valdes-Sanchez, L.; Relimpio, I.; Bhattacharya, S.S.; Quero, J.M. Electrostimulation in an autonomous culture lab-on-chip 
provides neuroprotection of a retinal explant from a retinitis pigmentosa mouse-model. Sensors and Actuators B: Chemical 2019, 288, 337-346.

11. Anastasova, S.; Kassanos, P.; Yang, G.Z. Multi-parametric rigid and flexible, low-cost, disposable sensing platforms for biomedical applications. Biosensors and Bioelectronics 2018, 102, 668-675.

12. Voeten, R.L.; Ventouri, I.K.; Haselberg, R.; Somsen, G.W. Capillary electrophoresis: trends and recent advances. Analytical chemistry 2018, 90, 1464-1481.

13. Mantovani, V.; Galeotti, F.; Maccari, F.; Volpi, N. Recent advances in capillary electrophoresis separation of monosaccharides, oligosaccharides, and polysaccharides. Electrophoresis 2018, 39, 179-189.

14. Hajba, L.; Guttman, A. Recent advances in column coatings for capillary electrophoresis of proteins. $\operatorname{Tr} A C$ Trends in Analytical Chemistry 2017, 90, 38-44.

15. Toraño, J.S.; Ramautar, R.; de Jong, G. Advances in capillary electrophoresis for the life sciences. Journal of Chromatography B 2019, 1118, 116-136.

16. de Carvalho, J.A.; Pinheiro, B.G.; Hagen, F.; Gonçalves, S.S.; Negroni, R.; Kano, R.; Bonifaz, A.; de Camargo, Z.P.; Rodrigues, A.M. A new duplex PCR assay for the rapid screening of mating-type idiomorphs of pathogenic Sporothrix species. Fungal Biology 2021.

17. Kaprou, G.D.; Papadopoulos, V.; Loukas, C.M.; Kokkoris, G.; Tserepi, A. Towards PCB-based miniaturized thermocyclers for DNA amplification. Micromachines 2020, 11, 258.

18. Kaprou, G.D.; Papadopoulos, V.; Papageorgiou, D.P.; Kefala, I.; Papadakis, G.; Gizeli, E.; Chatzandroulis, S.; Kokkoris, G.; Tserepi, A. Ultrafast, low-power, PCB manufacturable, continuous-flow microdevice for DNA amplification. Analytical and bioanalytical chemistry 2019, 411, 5297-5307.

19. Lee, P.Y.; Saraygord-Afshari, N.; Low, T.Y. The evolution of two-dimensional gel electrophoresis-from proteomics to emerging alternative applications. Journal of Chromatography A 2020, 1615, 460763.

20. Strobel, H.A.; Calamari, E.L.; Alphonse, B.; Hookway, T.A.; Rolle, M.W. Fabrication of custom agarose wells for cell seeding and tissue ring self-assembly using 3D-printed molds. JoVE (Journal of Visualized Experiments) 2018, p. e56618. 